\title{
F2 Gene
}

National Cancer Institute

\section{Source}

National Cancer Institute. F2 Gene. NCI Thesaurus. Code C82852.

This gene plays a role in the coagulation cascade. 